\title{
Elucidating Conceptual Properties from Word Embeddings
}

\author{
Kyoung-Rok Jang \\ School of Computing \\ KAIST \\ Daejeon, South Korea \\ kyoungrok.jang@kaist.ac.kr
}

\author{
Sung-Hyon Myaeng \\ School of Computing \\ KAIST \\ Daejeon, South Korea \\ myaeng@kaist.ac.kr
}

\begin{abstract}
In this paper, we introduce a method of identifying the components (i.e. dimensions) of word embeddings that strongly signifies properties of a word. By elucidating such properties hidden in word embeddings, we could make word embeddings more interpretable, and also could perform property-based meaning comparison. With the capability, we can answer questions like "To what degree a given word has the property cuteness?" or "In what perspective two words are similar?". We verify our method by examining how the strength of property-signifying components correlates with the degree of prototypicality of a target word.
\end{abstract}

\section{Introduction}

Modeling the meaning of words has long been studied and served as a basis for almost every kind of NLP tasks. Most recent word modeling techniques are based on neural networks, and the word representations produced by such techniques are called word embeddings, which are usually lowdimensional, dense vectors of continuous-valued components. Although word embeddings have been proved for their usefulness in many tasks, the question of what are represented in them is understudied.

Recent studies report empirical evidence that indicates word embeddings may reflect some property information of a target word (Erk, 2016; Levy et al., 2015). Learning the properties of a word would be helpful because many NLP tasks can be related to "finding words that possess similar properties", which include finding synonyms, named entity recognition (NER). Without a method for explicating what properties are contained in em- beddings, however, researchers have mostly focused on improving the performance in wellknown semantic benchmark tasks (e.g. SimLex999) as a way to find better embeddings.

Performing well in such benchmark tasks is valuable but provides little help in understanding the inside of the black box. For instance, it is not possible to answer to questions like "To what degree a given word has the property cuteness?".

One way to solve this problem is to elucidate properties that are encoded in word embeddings and associate them with task performances. With the capability, we can not only enhance our understanding of word embeddings but also make it easier to make comparisons among heterogeneous word embedding models in more coherent ways. Our immediate goal in this paper is to show the feasibility of explicating properties contained in word embeddings.

Our research can be seen as an attempt to increase the interpretability of word embeddings. It is in line with an attempt to provide a humanunderstandable explanation for complex machine learning models, with which we can gain enough confidence to use them in decision-making processes.

There has been a line of work devoted to identifying components that are important for performing various NLP tasks such as sentiment analysis or NER (Faruqui et al., 2015; Fyshe et al., 2015; Herbelot and Vecchi, 2015; Karpathy et al., 2016; Li et al., 2016a; Li et al., 2016b; Rothe et al., 2016). Those works are analogous to ours in that they try to inspect the role of the components in word embedding. However, they just attempt to identify key features for specific tasks rather than elucidating properties. In contrast, our question is "what comprises word embeddings?" not "what components are important for performing well in a specific task?" 


\section{Feasibility Study}

\subsection{Background}

Word embeddings can be seen as representing concepts of a word. As such, we attempt to design an property-related experiment around manipulation of concepts. In particular, we bring in the category theory (Murphy, 2004) where the notion of category is defined to be "grouping concepts that share similar properties". In other words, properties have a direct bearing on concepts and their categories, according to the theory.

On the other hand, researchers have argued that some concepts are more typical (or central) than others in a category (Rosch, 1973; Rosch, 1975). For instance, apple is more typical than olive in the fruit category. The typicality is a graded phenomenon, and may rise due to the strength of 'essential' properties that make a concept a specific category.

The key ideas from the above are 1) concepts of the same category share similar properties and 2) some concepts that have strong essential properties are considered more typical in specific category, and they guided our experiment design.

\subsection{Design}

The goal of this study is to show the feasibility of sifting property information from word embeddings. We assume that a concept's property information is captured and distributed over one or more components (dimensions) of embeddings during the learning process. Since the concepts that belong to the same category are likely to share similar properties, there should be some salient components that are shared among them. We call such components as SIG-PROPS (for significant properties) of a specific category.

In this feasibility study, we hypothesize that the strength of SIG-PROPS is strongly correlated with the degree of concept's typicality. This is based on the theory introduced in Section 2.1, that the typicality phenomenon rises due to the strength of essential properties a target concept possesses. So the concept that has (higher/lower) SIG-PROPS values than others should be (more typical/less typical) than other concepts.

\subsection{Datasets}

For our experiment dealing with typicality of concepts, we needed both (pre-trained) word embeddings and a dataset that encodes typicality scores of concepts to a set of categories. Below we describe two datasets we used in our experiment: HyperLex and Non-Negative Sparse Embedding (NNSE).

\subsubsection{Dataset: Non-Negative Sparse Embedding (NNSE)}

One desirable quality we wanted from the word embeddings to be used in our experiment is that there should be clear contrast between informative and non-informative components. In ordinary dense word embeddings, usually every component is filled with a non-zero value.

The Non-Negative Sparse Embedding (NNSE) (Murphy et al., 2012) fulfills the condition in the sense that insignificant components are set to zero. The NNSE component values falling between 0 and 1 (non-negative) are generated by applying the non-negative sparse coding algorithm (Hoyer, 2002) to ordinary word embeddings (e.g. word2vec).

\subsubsection{Dataset: HyperLex}

HyperLex is a dataset and evaluation resource that quantifies the extent of the semantic category membership (Vulić et al., 2016). A total of 2,616 concept pairs are included in the dataset, and the strength of category membership is given by native English speakers and recorded in graded manner. This graded category membership can be interpreted as a 'typicality score' (1-10). Some samples are shown in Table 1.

\begin{tabular}{|c|c|c|}
\hline Concept & Category & Score \\
\hline basketball & activity & 10 \\
\hline spy & agent & 8 \\
\hline handbag & bowl & 0 \\
\hline
\end{tabular}

Table 1: A HyperLex sample. The score is the answer to the question "To what degree is concept a type of category?"

\section{Experiment}

\subsection{Preparation}

We first prepared pre-trained NNSE embeddings. The authors released pre-trained model on their website $^{1}$. We used the model trained with dependency context ('Dependency model' on the website), because as reported in (Levy and Goldberg, 2014), models trained on dependency context tend

\footnotetext{
${ }^{1}$ http://www.cs.cmu.edu/ bmurphy/NNSE/
} 
to prefer functional similarity (hogwarts - sunnydale) rather than topical similarity (hogwarts dumbledore $)^{2}$. The embeddings are more sparse than ordinary embeddings and have 300 components.

Next we fetched HyperLex dataset at the author's website ${ }^{3}$. To make the settings suitable to our experiment goal, we selected categories with the following criteria:

1. The categories and instances must be concrete nouns (e.g. food). This is because people are more coherent in producing the properties of concrete nouns (Murphy, 2004). So the embeddings of concrete nouns should contain more clear property information than other types of words.

2. The categories must contain enough number of instances (not 1 or 2). This is to gain reliable result.

3. Some categories are sub-category of another selected category while others are not related. This is to see the discriminative and overlapping effect of identified SIG-PROPS between categories. Related categories should share a set of strong SIG-PROPS, while unrelated categories shouldn't.

As a result, we selected five categories: food, fruit (sub-category of food), animal, bird (subcategory of animal), and instrument. We fetched the concepts that belong to the categories and then filtered out those that aren't contained in the pretrained NNSE embeddings. The final size of each category is shown in Table 2 .

\begin{tabular}{|c|c|}
\hline Category & \# of Concepts \\
\hline food & 54 \\
\hline fruit & 9 \\
\hline animal & 46 \\
\hline bird & 16 \\
\hline instrument & 14 \\
\hline
\end{tabular}

Table 2: The size of selected categories

In the next section, we explain how we identified SIG-PROPS of each category.

\footnotetext{
${ }^{2}$ We thought "sharing similar function" is more compatible with the notion of sharing similar properties. The topical similarity is less indicative of having properties in common.

${ }^{3}$ http://people.ds.cam.ac.uk/iv250/hyperlex.html
}

\subsection{Identification of SIG-PROPS}

The goal of this step is to find SIG-PROPS that might represent each category. Simply put, SIGPROPS are the components that have on average high value compared to other components of the concepts in the same category. We identified SIGPROPS by 1) calculating an average value of each component across the concepts with the same category, then 2) choosing those components whose average value is above $h$. We empirically set $h$ to 0.2 .

\begin{tabular}{|c|c|c|}
\hline \multirow{2}{*}{ Category } & \multicolumn{2}{|c|}{ SIG-PROPS } \\
\cline { 2 - 3 } & Comp. ID & Avg. \\
\hline \multirow{2}{*}{ instrument } & c88 & 0.806 \\
\cline { 2 - 3 } & $\mathrm{c} 258$ & 0.769 \\
\hline \multirow{2}{*}{ animal } & $\mathbf{c 1 5 4}$ & 0.587 \\
\cline { 2 - 3 } & $\mathbf{c 2 6 5}$ & 0.221 \\
\hline \multirow{2}{*}{ bird } & $\mathbf{c 1 5 4}$ & 0.550 \\
\cline { 2 - 3 } food & $\mathbf{c 2 6 5}$ & 0.213 \\
\hline \multirow{3}{*}{ fruit } & $\mathrm{c} 207$ & 0.298 \\
\cline { 2 - 3 } & $\mathbf{c 2 3 3}$ & 0.269 \\
\hline & $\mathrm{c} 229$ & 0.492 \\
\cline { 2 - 3 } & $\mathrm{c} 27$ & 0.369 \\
\cline { 2 - 3 } & $\mathrm{c} 156$ & 0.349 \\
\cline { 2 - 3 } & $\mathrm{c} 44$ & 0.264 \\
\cline { 2 - 3 } & $\mathbf{c 2 3 3}$ & 0.206 \\
\hline
\end{tabular}

Table 3: SIG-PROPS of each category. The strings in "Comp. ID" column are the component IDs (c1-c300). "Avg." column indicates the average value of the component across all the concepts under that category.

Table 3 shows the identified SIG-PROPS. The number of SIG-PROPS is different across categories. Interestingly, there is component overlap between taxonomically similar categories ('c154' and 'c265' between animal-bird, 'c233' between food-fruit), while there is none between unrelated categories (instrument-animal-food).

This initial observation is encouraging for our feasibility study in that indeed SIG-PROPS can play a role of distinguishing or associating categories. We argue that the identified SIG-PROPS strongly characterize each category, showing that we can associate vector components with properties.

\subsection{Correlation between SIG-PROPS and concepts typicality scores}

In this section, we check how the strength of SIGPROPS correlates with the typicality scores. Note that the range of SIG-PROPS values differ from category to category - those for instrument are especially high, which might indicate they are highly 
representative.

Our assumption is that if the identified SIGPROPS truly represent the essential quality of a category, the strength of SIG-PROPS should be proportional to concepts' typicality scores (equation $1)$.

$$
\begin{array}{r}
\text { Strength(SIG-PROPS) } \\
\propto \text { Typicality (Concept) }
\end{array}
$$

We observe this phenomenon by calculating Pearson correlation between the strength of SIGPROPS and typicality scores. For instance, suppose we calculate the correlation between 'c88' (88th component) of instrument concepts and their typicality score. We inspect the instrument concepts one by one, collect their 'c88' values $(x)$ and typicality scores $(y)$, and then measure the tendency of changes in the two variables.

The result is shown in Table 4-8 where the column 'Rank' shows the rank of the component's correlation score (compared to other components). For instance, in Table 4 'c 88 ' has the highest correlation with the concept's typicality score.

\begin{tabular}{|c|c|c|}
\hline SIG-PROPS & Correlation & Corr. rank \\
\hline $\mathrm{c} 88$ & 0.926 & $1 \mathrm{st}$ \\
\hline $\mathrm{c} 258$ & 0.918 & $2 \mathrm{nd}$ \\
\hline
\end{tabular}

Table 4: Corr(SIG-PROPS, typicality): instrument

\begin{tabular}{|c|c|c|}
\hline SIG-PROPS & Correlation & Corr. rank \\
\hline c154 & 0.549 & 1 st \\
\hline c265 & 0.265 & $2 \mathrm{nd}$ \\
\hline
\end{tabular}

Table 5: Corr(SIG-PROPS, typicality): animal

\begin{tabular}{|c|c|c|}
\hline SIG-PROPS & Correlation & Corr. rank \\
\hline $\mathrm{c} 154$ & 0.783 & $1 \mathrm{st}$ \\
\hline $\mathrm{c} 265$ & 0.563 & $2 \mathrm{nd}$ \\
\hline
\end{tabular}

Table 6: Corr(SIG-PROPS, typicality): bird

\begin{tabular}{|c|c|c|}
\hline SIG-PROPS & Correlation & Corr. rank \\
\hline c233 & 0.255 & $1 \mathrm{st}$ \\
\hline $\mathrm{c} 120$ & 0.224 & $2 \mathrm{nd}$ \\
\hline $\mathrm{c} 207$ & 0.216 & 4 th \\
\hline $\mathrm{c} 192$ & 0.030 & $104 \mathrm{th}$ \\
\hline
\end{tabular}

Table 7: Corr(SIG-PROPS, typicality): food

\begin{tabular}{|c|c|c|}
\hline SIG-PROPS & Correlation & Corr. rank \\
\hline $\mathrm{c} 229$ & 0.743 & $1 \mathrm{st}$ \\
\hline $\mathrm{c} 233$ & 0.540 & 4 th \\
\hline $\mathrm{c} 27$ & 0.516 & 5 th \\
\hline $\mathrm{c} 44$ & 0.474 & 7 th \\
\hline $\mathrm{c} 156$ & -0.663 & 85th \\
\hline
\end{tabular}

Table 8: Corr(SIG-PROPS, typicality): fruit

As the results show, there is clear tendency SIGPROPS having high correlation with the typicality scores. Most of the SIG-PROPS showed meaningful correlation $(>0.5)$ with the typicality score or placed at the top in the component-typicality correlation ranking. The result strongly indicates that even when we apply the simple method of identifying SIG-PROPS and regarding them as properties, they serve as strong indicators for the concept's typicality.

\section{Conclusion and Future Work}

Although limited in scale, our work showed the feasibility of discovering properties from word embeddings. Not only SIG-PROPS can be used to increase the interpretability of word embeddings, but also enable us more elaborate, property-based meaning comparison.

Our next step would be checking the applicability to general NLP tasks (e.g. NER, synonym identification). Also, applying our method to word embeddings that have more granular components (e.g. 2,500) might be helpful for identifying SIGPROPS in more granular level.

\section{Acknowledgments}

This work was supported by Institute for Information \& communications Technology Promotion(IITP) grant funded by the Korea government(MSIP) (No. R0126-16-1002, Development of agro-livestock cloud and application service for balanced production, transparent distribution and safe consumption based on GS1)

\section{References}

Katrin Erk. 2016. What do you know about an alligator when you know the company it keeps? Semantics and Pragmatics Article, 9(17):1-63.

Manaal Faruqui, Yulia Tsvetkov, Dani Yogatama, Chris Dyer, and Noah A. Smith. 2015. Sparse overcomplete word vector representations. In Proceedings of the 53rd Annual Meeting of the Association for Computational Linguistics and the 7th International 
Joint Conference on Natural Language Processing (Volume 1: Long Papers), pages 1491-1500, Beijing, China, July.

Alona Fyshe, Leila Wehbe, Partha P. Talukdar, Brian Murphy, and Tom M. Mitchell. 2015. A compositional and interpretable semantic space. In Proceedings of the 2015 Conference of the North American Chapter of the Association for Computational Linguistics: Human Language Technologies, pages 3241, Denver, Colorado, May-June.

Aurélie Herbelot and Eva Maria Vecchi. 2015. Building a shared world: Mapping distributional to model-theoretic semantic spaces. In Proceedings of EMNLP, number September, pages 22-32, Lisbon, Portugal.

P. O. Hoyer. 2002. Non-negative sparse coding. In Neural Networks for Signal Processing - Proceedings of the IEEE Workshop, volume 2002-Janua, pages 557-565.

Andrej Karpathy, Justin Johnson, and Li Fei-Fei. 2016. Visualizing and Understanding Recurrent Networks. International Conference on Learning Representations (ICLR), pages 1-13.

Omer Levy and Yoav Goldberg. 2014. Dependencybased word embeddings. In Proceedings of the 52nd Annual Meeting of the Association for Computational Linguistics (Volume 2: Short Papers), pages 302-308, Baltimore, Maryland, June.

Omer Levy, Steffen Remus, Chris Biemann, and Ido Dagan. 2015. Do supervised distributional methods really learn lexical inference relations? In Proceedings of the 2015 Conference of the North American Chapter of the Association for Computational Linguistics: Human Language Technologies, pages 970-976, Denver, Colorado, May-June.

Jiwei Li, Xinlei Chen, Eduard Hovy, and Dan Jurafsky. 2016a. Visualizing and Understanding Neural Models in NLP. In Naacl, pages 1-10.

Jiwei Li, Will Monroe, and Dan Jurafsky. 2016b. Understanding Neural Networks through Representation Erasure. In Arxiv.

Brian Murphy, Partha Pratim Talukdar, and Tom Mitchell. 2012. Learning Effective and Interpretable Semantic Models using Non-Negative Sparse Embedding. In Proceedings of COLING 2012: Technical Papers, number December 2012, pages 1933-1950.

Gregory Murphy. 2004. The big book of concepts. MIT press.

Eleanor H. Rosch. 1973. Natural categories. Cognitive Psychology, 4(3):328-350.

Eleanor H. Rosch. 1975. Cognitive representations of semantic categories. Journal of experimental psychology: General, 104(3):192.
Sascha Rothe, Sebastian Ebert, and Hinrich Schütze. 2016. Ultradense word embeddings by orthogonal transformation. In Proceedings of the 2016 Conference of the North American Chapter of the Association for Computational Linguistics: Human Language Technologies, pages 767-777, San Diego, California, June.

Ivan Vulić, Daniela Gerz, Douwe Kiela, Felix Hill, and Anna Korhonen. 2016. HyperLex: A Large-Scale Evaluation of Graded Lexical Entailment. Arxiv. 